\title{
Synthesis and characterization of ordered mesoporous silica nanoparticles with tunable physical properties by varying molar composition of reagents
}

\author{
Harrison Wanyika ${ }^{1,3 *}$, Erastus Gatebe ${ }^{1}$, Paul Kioni ${ }^{2}$, Zhiyong Tang ${ }^{3}$ and Yan Gao ${ }^{3}$ \\ ${ }^{1}$ Department of Chemistry, Jomo Kenyatta University of Agriculture and Technology, 62 000-00200 Nairobi, Kenya. \\ ${ }^{2}$ Kimathi University College of Technology, 657-10100 Nyeri, Kenya. \\ ${ }^{3}$ National Centre for Nanoscience and Technology, Beijing 100190, P.R. China.
}

Accepted 22 November, 2011

\begin{abstract}
Mesoporous silica nanoparticles (MSN) with tunable physical and surface properties would find application in various biotechnological and biomedical fields. In this study, a series of MSN with varied physical properties were synthesized via liquid crystal templating (LCT) mechanism by varying the molar concentrations of the reagents. Characterization of the prepared materials was done by scanning electron microscopy (SEM), transmission electron microscopy (TEM), nitrogen adsorption/desorption isotherms, Fourier transform infrared (FT-IR) spectroscopy, X-ray diffraction (XRD) spectroscopy, thermal gravimetric analysis (TGA) and differential thermal analysis (DTA). The particle sizes, BarrettJoyner-Halenda (BJH) pore sizes, Brunauer-Emmett-Teller (BET) surface areas and BJH total pore volume were tuned between 50 to $900 \mathrm{~nm}, 2.4$ to $4.4 \mathrm{~nm}, 589$ to $1163 \mathrm{~m}^{2} \mathrm{~g}^{-1}$ and 0.61 to $0.83 \mathrm{~cm}^{3} \mathrm{~g}^{-1}$, respectively. The effects of reagents concentrations in the variation of the properties were discussed. The study demonstrated the versatility of the liquid-based synthesis method in the preparation of MSN with different physical properties.
\end{abstract}

Key words: Mesoporous silica nanoparticles, physical properties, tuning, synthesis.

\section{INTRODUCTION}

Mesoporous silicas, such as MCM-41 and SBA-15 silicas, are solid materials, which are comprised of a honeycomb-like porous structure with hundreds of empty channels. Recently, mesoporous silica nanoparticles (MSN) have been intensively explored in materials research due to their unique properties, such as high surface areas, large pore volumes, tunable pore sizes with a narrow distribution and tunable particle diameters (Bottini et al., 2007; Gerion et al., 2007). Zhu et al. (2007) demonstrated the biocompatiblity, the high structural stability and chemical versatility of silica using silicacoated semiconductor quantum dots. Furthermore, the biodegradability of silica particles has been shown in mice. Popat et al. (2011) used fluorescent labeled silica particles at a low dose of $20 \mathrm{mgkg}^{-1}$, the particles were

\footnotetext{
*Corresponding author. E-mail: hwanyika@gmail.com. Tel: +254-67-52181. Fax: +254-67-52164.
}

cleared from the mice body through the renal system. Additionally, the researchers noted that MSN are nontoxic at relatively low doses.

MSN with controlled properties can be extended to a wide range of applications, such as, drug delivery, catalysts supports, adsorption and separation of proteins, cell imaging, cell labeling, enzyme adsorption and immobilezation (Cauda et al., 2009). More specifically, the potential utilization of MSN materials in medical and pharmaceutical drug delivery systems is well documented (Courtney et al., 2010; Speybroeck et al., 2010; ValletRegi, 2009; Popovici et al., 2010; Leirose and Cardoso, 2011; Limnel et al., 2011; Zhu et al., 2011; Shen et al., 2011; Vivero-Escoto et al., 2010). Radin et al. (2004) research findings showed that MSN could be used to store and gradually release antibiotics and other drugs since they posses' abundant pore surface $\mathrm{Si}-\mathrm{OH}$ bonds for loading and releasing drug molecules. Moreover, the use of nanoparticles as drug carriers can enhance penetration of therapeutic drugs into the brain (Cui et al., 2009) 
and prolong blood circulation time of drugs (Shokri et al., 2011). The suitability of MSN for important biotechnological and biomedical applications is also due to their small size which allows a facile endocytosis by living animal and plant cells without any significant cytotoxicity. Small and tunable pore sizes allow the adjustment of loading of different drug molecules and to study the kinetics of drug release with high precision. High surface areas and large pore volumes allows high loading, while the unique porous structure renders the "no-leaking" capability of loaded drug molecules (Popat et al., 2011; Radin et al., 2004).

MSN of various types and exhibiting different properties have previously been synthesized via different methods (Igor et al., 2008; Gu et al., 2007; Slowing et al., 2007; Naik et al., 2007; Yoo and Stein, 2011; Niu et al., 2010; Borisova et al., 2011; Suteewong et al., 2011; Yokoi et al., 2006; Mbaraka et al., 2006; Graf et al., 2006). In MSN synthesis, various chemical materials, such as sodium silicate, tetramethyl ammonium silicate and tetraethyl orthosilicate etc. are used as silica sources. Quar-ternary ammonium surfactants are used as structure-directing agents under a wide range of $\mathrm{pH}$ and temperature conditions (Gao et al., 2006; Liu et al., 2011). By liquid crystal templating (LCT) mechanism, in aqueous solution, surfactant micelles organize into an ordered array of hexagonal micellar rods that form a liquid template. Hydrolysis of the silica precursor yields the silica nucleus which enters the template. Inorganic silica walls are formed upon condensation of the silicate seeds (Hoffman et al., 2006). The template is subsequently removed by either solvent extraction using alcohols (methanol or ethanol) or by calcination at high temperatures. Calcination is a key step for obtaining accessible mesopores, large surface areas and pore volumes. During calcination, condensation of silanol groups $(\mathrm{Si}-\mathrm{OH})$ into siloxane ( $\mathrm{Si}-\mathrm{O}-\mathrm{Si}$ ) bridges takes place, thus consolidating the mesoporous structure (Cauda et al., 2011).

Other models, such as the micelle assembly model, suggests that, inorganic species interacts with micelles in solution and mediate an organization process leading to liquid-crystal like structures while the general co-operative assembly model argued that the 1st stage of the process was multidentate binding of silica species to the surfactant, followed by co-operative assembly of a liquid crystal like phase, driven by the necessity for charge density matching between charged silica and surfactant (Kobler and Bein, 2008).

Researchers are incessantly engaged in efforts to come up with facile methods for the synthesis of MSN with controlled properties, mostly, smaller particle sizes, tunable pore diameters, high surface area and high pore volumes. The use of auxiliary reagents, such as pore expanders and/or use of surfactants with varying chain lengths are the commonly used techniques for tuning MSN properties. However, varying the ratio of reagents concentration in a typical reaction mixture to achieve the same or better results would be simpler and cheaper.
The current study focused on the manipulation of physical properties of various MSN products by varying the concentration of the reagents while maintaining all the other factors constant. Synthesis was carried out using cetyltrimethylammonium bromide (CTAB) as a structure directing agent and tetraethyl orthosilicate (TEOS) as a silica precursor in alkaline aqueous media. Our research findings show the dynamism of reagents concentrations in achieving different MSN properties. A thorough discussion of the role of various reagents in tuning the properties of MSN was done to augment experimental results.

\section{MATERIALS AND METHODS}

All the chemicals used in this study were of analytical grade. CTAB, TEOS $\left(\mathrm{SiO}_{2}>28.0 \%\right)$ and sodium hydroxide (>96\%) were purchased from Sigma-Aldrich, UK. All the reagents were used as purchased without further purification. Water used in the experiments was purified by Millipore Milli-Q system to a resistivity of $18.2 \mathrm{~m} \Omega . \mathrm{cm}$.

\section{Synthesis of mesoporous silica nanoparticles}

Various samples were prepared from reaction mixtures with different concentrations of reagents as outlined in Table 1. CTAB was dissolved in water. Aqueous $\mathrm{NaOH}(2.0 \mathrm{M})$ was added to the CTAB solution, and the solution temperature was adjusted to $80^{\circ} \mathrm{C}$ in a silicon oil bath with stirring using a magnetic stirrer at 800 rotations per minute. The solution was heated for 30 min after which TEOS was added dropwise, and the mixture was allowed to stir for $2 \mathrm{~h}$ at $80^{\circ} \mathrm{C}$. The resultant white precipitate was isolated by vacuum filtration using a Buckner funnel and was washed several times with copious amount of water. The product was dried in an oven for $2 \mathrm{~h}$ at $140^{\circ} \mathrm{C}$ and ground into powder using a pestle and a mortar. Calcination of the material was performed at $550^{\circ} \mathrm{C}$ for 5 $\mathrm{h}$ to remove the template.

\section{Characterization}

The morphology and size of the prepared samples was characterized by scanning electron microscope (SEM) model, Hitachi S-4800 and transmission electron microscope (TEM) model, Tecnai G ${ }^{2} 20$ S-TWIN. Sample preparation was done by dispersing a little amount of sample in water. A thin film of the homogenous sample solution was deposited and air dried on the reflective surfaces of silicon wafer for SEM analysis. The sample was casted on carbon coated copper grid for TEM analysis. Based on Electron microscopy results, further characterization was done only for the first five MSN (a to e).

Nitrogen adsorption/desorption isotherms were measured by ASAP 2020 Micromeritics surface area analyzer at $-196^{\circ} \mathrm{C}$. The specific surface areas were assessed according to the standard BrunauerEmmett-Teller (BET) method (Brunauer et al., 1938). The pore size distributions and total pore volume were determined from adsorption branches of isotherms by the Barrett-Joyner-Halenda (BJH) method (Barrett et al., 1951). Fourier transform infrared (FT-IR) analysis was carried out using $\mathrm{KBr}$ discs in the 400 to $4000 \mathrm{~cm}^{-1}$ range by Perkin Elmer Spectrometer (SPECTMM ONE B). The powder X-ray diffraction (XRD) patterns were obtained with an X'PertPro PANalytical, LR 39487C XRD diffractometer using $\mathrm{Cu} \mathrm{Ka}$ radiation $(40 \mathrm{kV}, 40 \mathrm{~mA})$. Stepwise increase for small angle was $0.01^{\circ} \mathrm{s}^{-1}$ over the range of 1 to $8^{\circ}$ and wide angle rate of $1^{\circ} 2 \theta \mathrm{min}^{-1}$ over the range of 8 to $90^{\circ} 2 \theta$, respecttively. Samples were prepared as thin layers on glass slides. Thermal gravimetric analysis (TGA) and differential thermal analysis (DTA) were done on a Perkin-Elmer thermal gravimetric analyzer (TGA-7) from 
Table 1. Reagents compositions for MSN synthesis.

\begin{tabular}{lcccc}
\hline \multirow{2}{*}{ Sample code e } & \multicolumn{4}{c}{ Composition } \\
\cline { 2 - 5 } & CTAB (mM) & TEOS (mM) & NaOH (mM) & $\mathbf{H}_{2} \mathbf{O}$ (moles) \\
\hline MSN - 1 (a) & 2.74 & 27.09 & 7.0 & 21.11 \\
MSN - 2 (b) & 2.74 & 27.09 & 7.0 & 26.67 \\
MSN - 3 (c) & 2.74 & 27.09 & 14.0 & 26.67 \\
MSN - 4 (d) & 2.74 & 27.09 & 3.8 & 21.11 \\
MSN - 5 (e) & 2.74 & 27.09 & 7.6 & 42.22 \\
MSN - 6 (f) & 2.74 & 13.54 & 7.0 & 21.11 \\
MSN - 7 (g) & 2.74 & 54.17 & 7.0 & 21.11 \\
MSN - 8 (h) & 1.37 & 27.09 & 7.0 & 21.11 \\
MSN - 9 (i) & 5.49 & 27.09 & 7.0 & 21.11 \\
MSN - 10(j) & 5.49 & 27.09 & 7.0 & 10.55 \\
MSN - 11(k) & 2.74 & 27.09 & 14.0 & 21.11 \\
\hline
\end{tabular}

${ }^{*} \mathrm{mM}$ - millimoles

from $\mathrm{RT}$ to $800^{\circ} \mathrm{C}$ at the heating rate of $5^{\circ} \mathrm{C} \min ^{-1}$ in air.

\section{RESULTS AND DISCUSSION}

\section{Microscopy observations}

SEM images disclose various particle sizes and morphologies (Figure 1). Corresponding TEM images show regular hexagonal arrays of channels (Figure 2), which confirmed the honeycomb like structure of MSN. This was in good accordance with the expectations based on the work of previous researchers (Kobler et al., 2008; Stober et al., 1968).

Spherical nanoparticles (NPs) with narrow particle size distribution of $\sim 150 \mathrm{~nm}$ in average diameter were obtained for MSN-1 (Figure 1a). However, when the $\mathrm{NaOH}$ concentration was reduced to $3.8 \mathrm{mM}$ (MSN-4), NPs with uniform size distribution of $\sim 50 \mathrm{~nm}$ were obtained (Figure 1d). Increase of the concentration of $\mathrm{NaOH}$ to $7.6 \mathrm{mM}$ coupled with double increase of water to 42.22 moles (MSN-5), resulted in the formation of $\sim 100 \mathrm{~nm}$ nanospheres (Figure 1e). Use of 26.67 moles of water (MSN2 ), led to $200 \mathrm{~nm}$ sized particles (Figure 1b). SEM micrograph in Figure 1c corresponds to a scenario where the concentration of $\mathrm{NaOH}$ was increased two fold to $14.0 \mathrm{mM}$ and $\mathrm{H}_{2} \mathrm{O}$ increased to 26.67 moles (MSN-3), the results show aggregated particles of $\sim 900 \mathrm{~nm}$. When the concentration of $\mathrm{NaOH}$ was doubled without correspondding increase in the amount of solvent (MSN-11), many particles agglomerated, although few smaller spheres were also formed. Reduction of the concentration of TEOS to $13.54 \mathrm{mM}$ (MSN-6), also led to aggregation of particles and poor mesopore channels (Figure $1 \mathrm{f}$ and Figure 2f). Further tests revealed that, doubling TEOS concentration (MSN-7), led to formation of grey colored microparticles. Attempts to half the concentration of
CTAB (MSN-8), led to greyish product after calcination. Similarly, no precipitation of the product took place under the high CTAB concentration (MSN-9). Additionally, reducing the concentration of water to 10.55 moles (MSN-10) yielded no siliceous material.

The relatively low concentration of $\mathrm{NaOH}$ used to achieve small monodispersed particles in MSN-4 suggested that the concentration of the morphology catalyst/ structure stabilizer had to be kept in a certain ratio with respect to other reagents for high-quality nanoparticles, that is, those with a defined diameter, monodisperse size distribution, and small degree or no agglomeration to be realized. Various methods have been reported in literature to be effective in the control of particle size and agglomeration behavior of nanoparticles in liquid-phase synthesis. High dilution as it was in MSN-5 has been reported to control nucleus growth and agglomeration by complete separation of the reaction spaces (Feldmann and Goesmann, 2010). Furthermore, high water concentration is absolutely important for complete hydrolysis of TEOS $\left(\mathrm{Si}(\mathrm{OR})_{4}, \mathrm{R}=\mathrm{C}_{2} \mathrm{H}_{5}\right)$, this ensures that all of the OR groups are replaced by $\mathrm{OH}$ group, that is, $\mathrm{Si}(\mathrm{OR})_{4}+4$ $\mathrm{H}_{2} \mathrm{O} \rightarrow \mathrm{Si}(\mathrm{OH})_{4}+4 \mathrm{R}-\mathrm{OH}$. The cause for the bigger particles in MSN-3 and MSN-11 was attributed to higher particle growth rate than the nucleation rate as a result of a faster reaction since hydrolysis of the precursor and condensation of nanoparticles is supported by the high concentration of the catalyst. These findings agree well with previous work by Kobler and Bein (2008). Previous research by Wen et al. (2005) attributed particle aggregation to siloxane bond formation between free hydroxyl groups. Aggregation is always energetically favored over nanoparticles since it minimizes surface areas and saturates the bonding and co-ordination sites and therefore, in order to prevent the nanoparticles from further growth or aggregation, the particle surfaces should be saturated immediately after nucleation by electrostatic or steric 

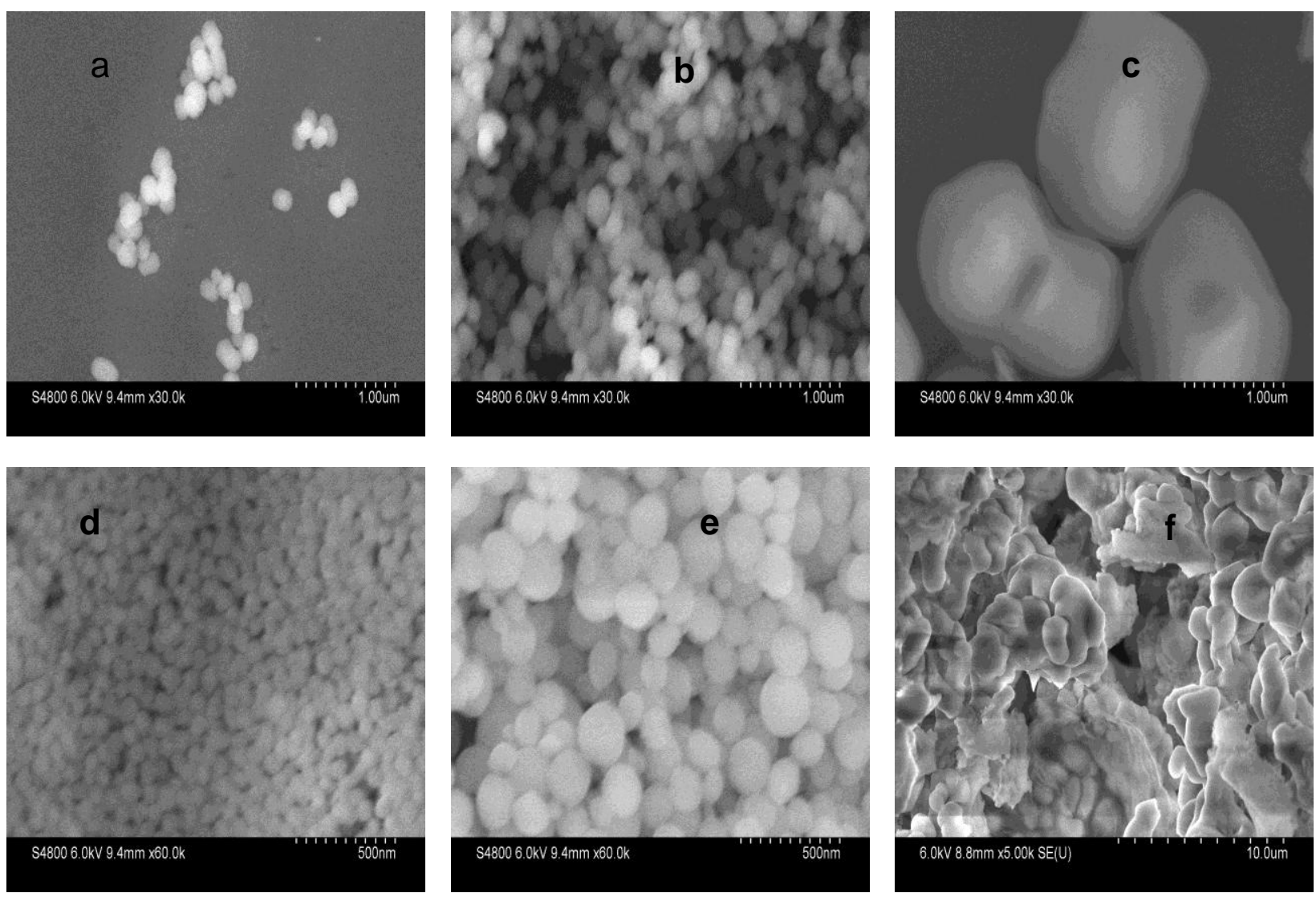

Figure 1. SEM images of MSN-1 (a), MSN-2 (b), MSN-3 (c), MSN-4 (d), MSN-5 (e) and MSN-6 (f). Various particle sizes and morphology are exhibited.

stabilization. According to Stober et al. (1968), the precursor concentration influences both the growth of seeds and the nucleation rate and thus, the number of initial seeds. The amount of precursor added in a synthesis is of paramount significance, thus, it has to be kept in a certain range which would guarantee high final product yield and desirable particle sizes. Very high concentration of the precursor may cause its incomplete hydrolysis leading to reagent residue in the product which explains the greying effect observed after calcinations when TEOS concentration was doubled.

The reason for formation of different products with different concentrations of CTAB is because particle formation was templated by micelles, as it is well exemplified in literature (Beck et al., 1992). At surfactant concentration, below the critical micelle concentration, surfactants do not aggregate into micelles, and thus, the low CTAB concentration situation was template deficient. Although, high concentration of surfactant is usually associated with more order in a LCT synthesis, a proper balance has to be struck between it and the other reagents in order to obtain ordered mesostructures.

In the study of the effects of surfactant concentration on the regularity and morphology of mesoporous silica,
Coleman and Attard (2001) found out that if the concentration of other reagents is fixed, there is an optimal concentration of surfactant below or above which order is lost. Palmqvist (2003) observed that, a balance of the molar concentration of all the reagents in the reaction mixture is absolutely necessary for formation of meso-structure. Previous research show that increasing the concentration of CTAB and TEOS decreases the particle size (Nooney et al., 2002); however, the negative findings in the current study suggest that the highest concentra-tions of the surfactant and the silica precursor exceeded the optimum concentrations required for the formation of mesoporous silica structures. The observation that no siliceous material was formed with low water concentration is well supported by literature which indi-cates that mesoporous silica nanoparticles assemble in highly dilute aqueous solutions (Feldmann and Goesmann, 2010).

It was revealed that the particle order, size and morphology of MSN can be tuned by varying the con-centration of the reagents in a typical reaction mixture. This observation is in good agreement with the nature of mesoporous silica nanoparticles as reported in literature (Beck et al., 1992). 

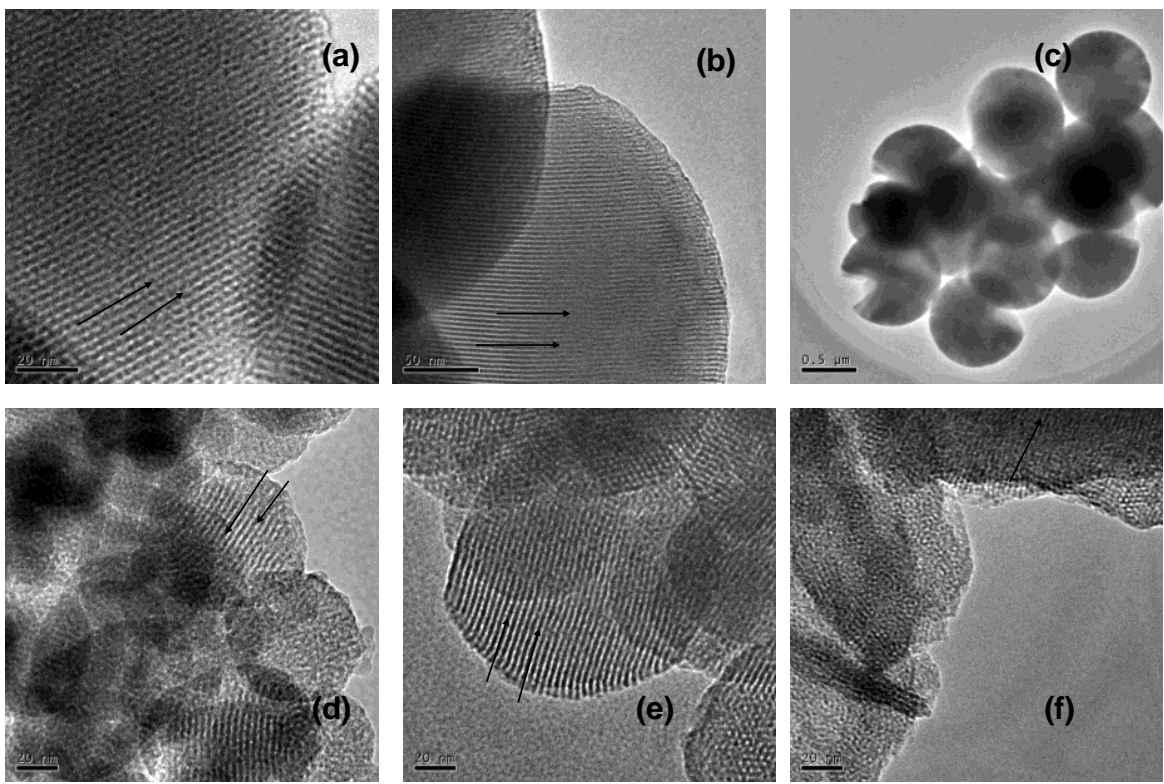

Figure 2. TEM images of MSN-1 (a), MSN-2 (b), MSN-3 (c), MSN-4 (d), MSN-5 (e) and MSN-6 (f). Honeycomb structure is demonstrated. Arrows indicate the position of channels.

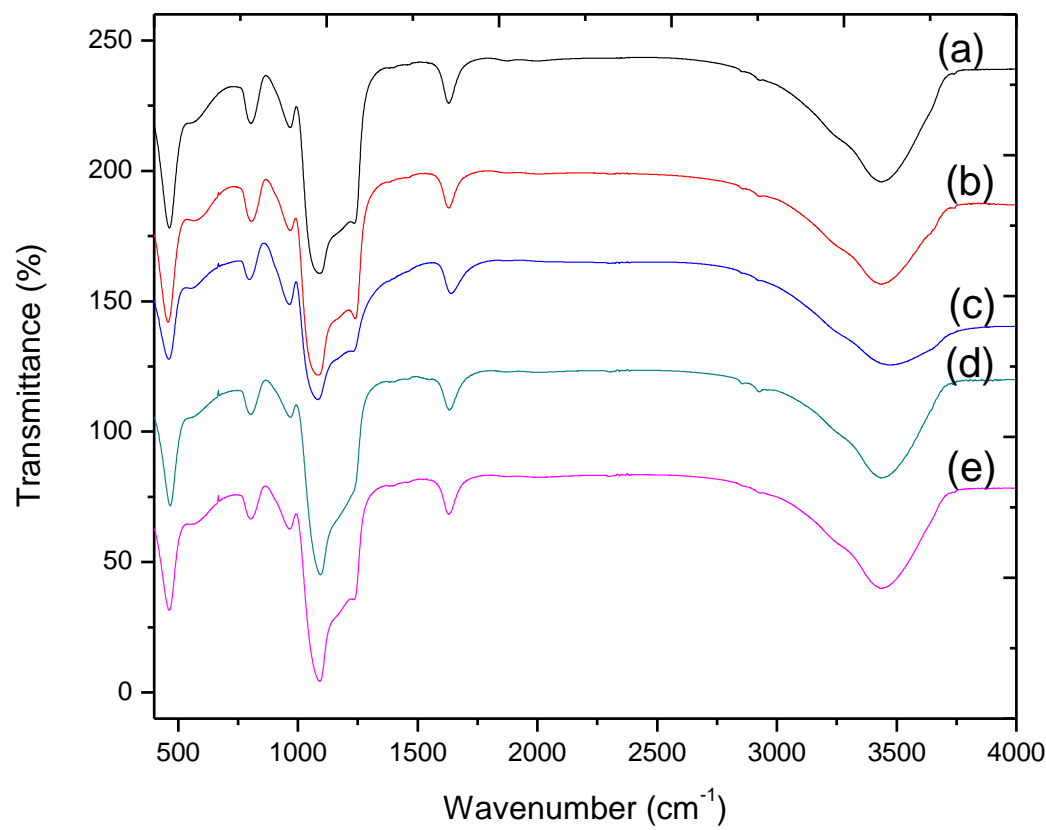

Figure 3. FT-IR spectra of MSN. Characteristic vibrational modes of typical MSN materials are exhibited in all the cases.

\section{FT-IR spectra}

The FT-IR spectra of MSN are displayed in Figure 3. Comparisons of the spectra show that they exhibit similar vibrational bands characteristic of mesoporous silica network. The broad band at $3435 \mathrm{~cm}^{-1}$ was attributed to the stretching vibration of the $\mathrm{Si}-\mathrm{OH}$ groups, the strong $1100 \mathrm{~cm}^{-1}$ bands were ascribed to Si-O-Si stretching vibrations, while the $1628,970,804$ and $460 \mathrm{~cm}^{-1}$ were attributed to $\mathrm{SiO}-\mathrm{H}$ bending, Si-OH bending, SiO-H symmetrical stretching and $\mathrm{Si}-\mathrm{O}$ bending vibrations, respectively. Similar patterns have been reported by other 


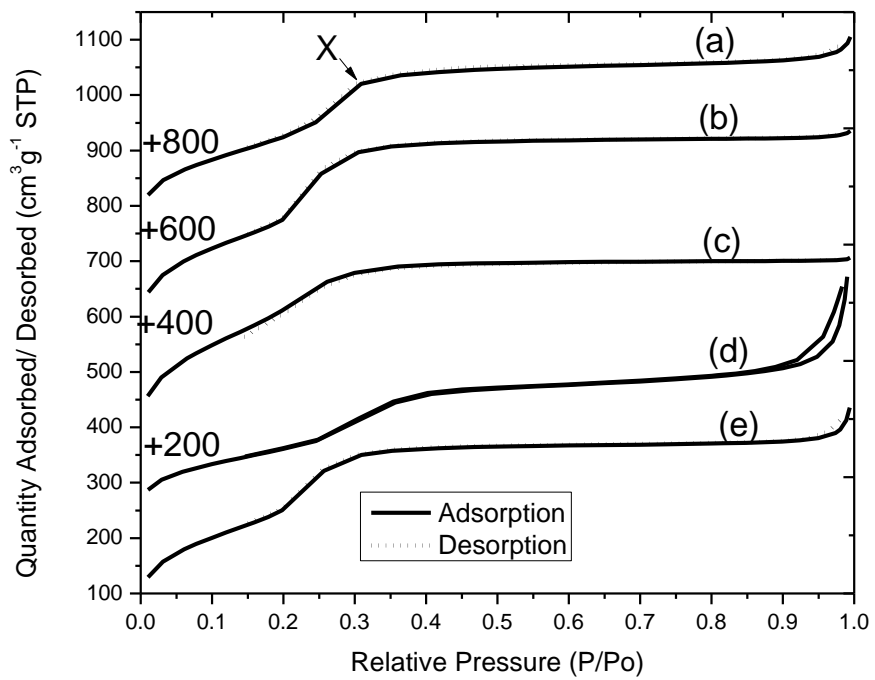

Figure 4. Nitrogen adsorption/desorption isotherms of MSN. Type IV isotherms typical of mesoporous silica are exhibited.

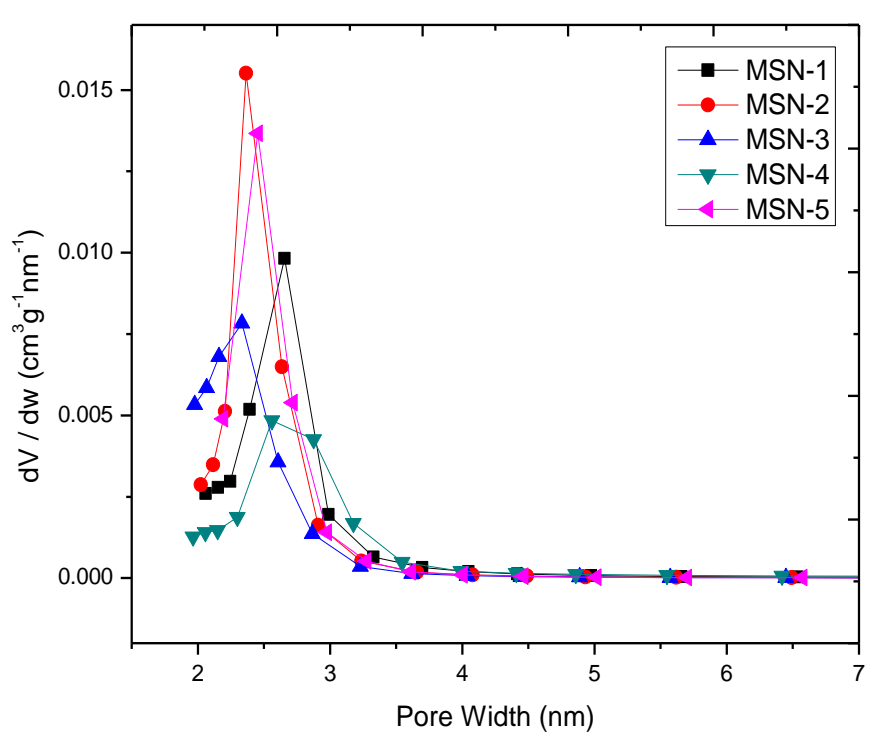

Figure 5. BJH narrow pore size distributions of MSN (based on adsorption branch).

researchers (Zhao et al., 1997; Salonen et al., 1997).

\section{Nitrogen adsorption/desorption studies}

Nitrogen adsorption/desorption isotherms of MSN are presented in Figure 4. Three distinct adsorption/desorption sections corresponding to relative pressures of $<0.3$, $0.3-0.9$ and $>0.9$ are notable. Small and narrow hysteresis loops are present at desorption sections corresponding to high relative pressure values. The sorption studies yielded type IV isotherms, characteristic of mesoporous materials (Sing et al., 1985). The hysteresis loops observed were associated with capillary condensation taking place in mesopores. Point $X$ (Figure $4 a$ ) indicates the stage at which monolayer coverage is complete and multilayer adsorption about to begin. BJH pore size distributions are given in Figure 5, while $\mathrm{BJH}$ total pore volumes and BET surface areas are shown in Table 2. Narrow pore size distributions were realized in all the samples. Average pore diameters ranged from $2.4 \mathrm{~nm}$ for MSN-3 to $4.4 \mathrm{~nm}$ for MSN-4 which was attributed to variation in the catalyst concentration. Besides promoting aggregation of particles, very high concentration of $\mathrm{NaOH}$ catalyst, generated small pore sizes, while the converse was true. The smallest pore volume was obtained with MSN-3, while the largest was obtained with MSN-2, again emphasizing the fact that, an optimal balance of composition of reagents is critical in obtaining quality products, that is, those that generally meet the threshold of all the properties desired. The utilized method for the determination of pore volumes and pore size distributions assumes cylindrical pores and thus, underestimates the true pore size (Thommes et al., 2000). Contrary to our expectation, the $\sim 900 \mathrm{~nm}$ MSN revealed the highest BET surface area, while the $\sim 50 \mathrm{~nm}$ MSN depicted the lowest. However, direct correlation between surface areas and particle sizes was not observed in all the cases. This confirmed that, MSN with a combination of different physical properties can be achieved by varying reactant ratios in any manner. For example, MSN-2 posses 200 $\mathrm{nm}$ relatively monodisperse spheres, very small pore size, very high surface area and very high pore volume, whereas, MSN-4 has 50 nm particles, monodispersed, high pore volume but bigger pore size and smaller surface area.

\section{XRD patterns}

The small angle X-ray diffraction (SAXRD) patterns of the calcined samples (Figure 6A) show three well-defined peaks corresponding to 100,110 and $200 \mathrm{hkl}$ miller indices, which indicated two dimensional (2D) long-range ordering and a hexagonal pore structure. The absence of the 110 and 200 peaks in MSN-3 and MSN-4 indicated that the mesopores of the two MSN products were less ordered. This was corroborated visually by TEM images in Figure 2. It was evident that very high or low concentration of $\mathrm{NaOH}$ led to low degree of mesopore ordering, which implied that a balance has to be kept between optimum concentration of the basic catalyst/structure stabilizer and other reagents. Wide angle X-ray diffraction (WAXRD) show a strong diffraction peak in the range of 17 to $30^{\circ} 2 \theta$ (Figure 6B), which unveiled regular periodic variations of the electronic density due to the long-range ordering of the pores in the material (Chen 
Table 2. Physical properties of MSN. Particle diameters were estimated from SEM and TEM images.

\begin{tabular}{|c|c|c|c|c|}
\hline Sample & $\begin{array}{c}\text { Average particle diameter } \\
(\mathrm{nm})\end{array}$ & Average pore diameter $(\mathrm{nm})^{\mathrm{a}}$ & $\begin{array}{c}\text { Surface area } \\
\left(\mathrm{m}^{2} / \mathrm{g}\right)^{\mathrm{b}}\end{array}$ & $\begin{array}{l}\text { Total pore volume } \\
\left(\mathrm{cm}^{3} / \mathrm{g}\right)^{\mathrm{c}}\end{array}$ \\
\hline MSN-1 (a) & 150 & 2.9 & 825 & 0.74 \\
\hline MSN-2 (b) & 200 & 2.5 & 1020 & 0.83 \\
\hline MSN-3 (c) & 900 & 2.4 & 1163 & 0.61 \\
\hline MSN-4 (d) & 50 & 4.4 & 589 & 0.79 \\
\hline MSN-5 (e) & 100 & 2.9 & 928 & 0.81 \\
\hline
\end{tabular}

${ }^{\mathrm{a}} \mathrm{BJH}$ average pore diameter. ${ }^{\mathrm{b}} \mathrm{BET}$ surface area. ${ }^{\mathrm{c}} \mathrm{BJH}$ total pore volume.
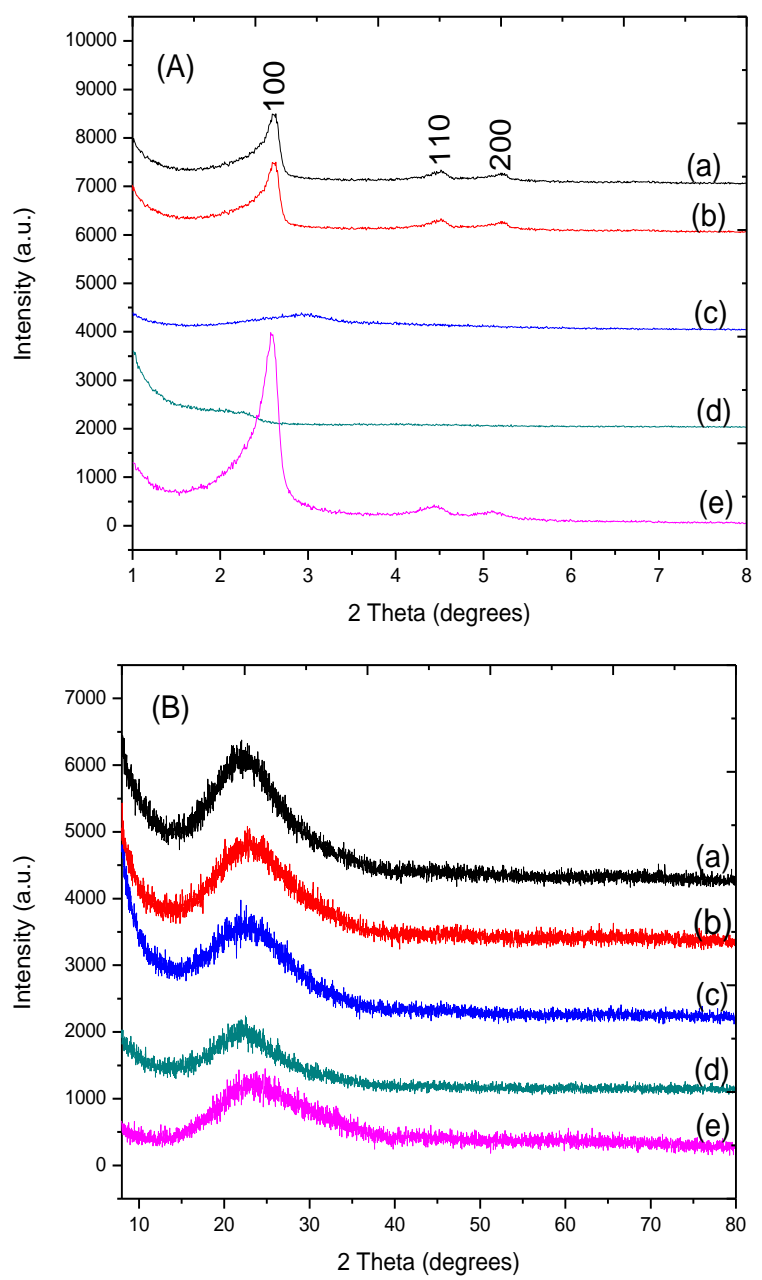

Figure 6. Powder SAXRD (A) and WAXRD (B) patterns of MSN. Typical patterns of hexagonal mesoporous silica are shown.

et al., 2011).

\section{TGA/DTA curves}

Thermal analysis results for MSN are as shown in Figure
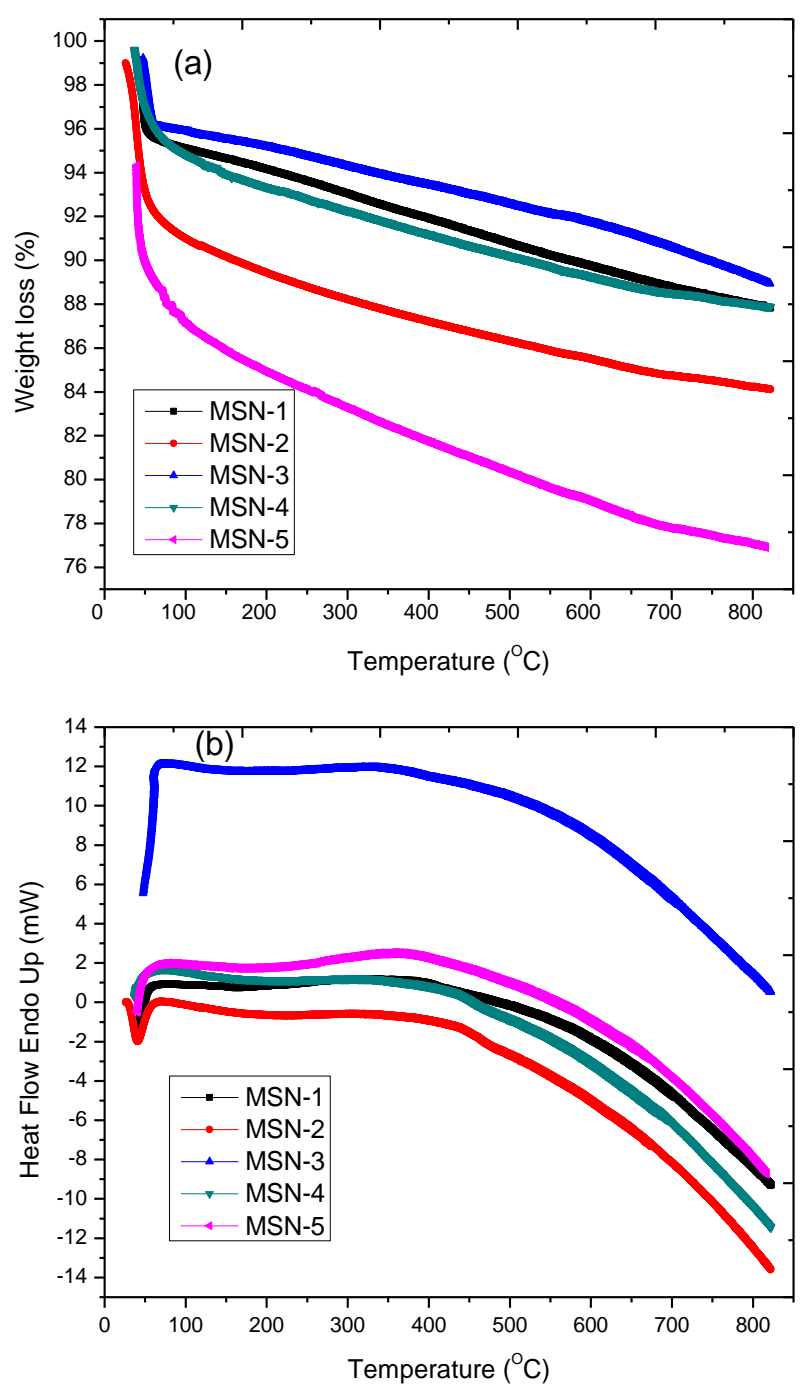

Figure 7. TGA (a) and corresponding DTA (b) curves for calcined MSN. They reveal thermal stability of MSN.

7. TGA curves depicted some little weight loss at low temperature which was attributed to loss of adsorbed water. Indeed, this was supported by the presence of 
endothermic peaks at $\angle 100^{\circ} \mathrm{C}$ in the DTA curves. Loss in weight at higher temperature was ascribed to loss of surface silanol groups. There was no significant weight loss due to disintegration of MSN which implied that the MSN were thermally stable.

\section{Conclusion}

The synthesis resulted in MSN with varied particle diameters and different degrees of aggregation. BJH pore sizes, BET surface areas and BJH total pore volumes of the various MSN also differed. Degrees of mesopore order of the various products were also found to be different. It was established that a delicate balance between all the reagents in the reaction mixture is necessary in order to synthesize nanoparticles with desirable properties. Our research findings show that, Liquid-based MSN synthesis methods are versatile and robust. The simple and cheap synthetic logic can be employed to prepare siliceous nanoparticles with different properties.

\section{ACKNOWLEDGEMENTS}

This work was supported by the Third World Academy of Sciences (TWAS), Chinese Academy of Sciences (CAS) and Jomo Kenyatta University of Agriculture and Technology (JKUAT).

\section{REFERENCES}

Barrett EP, Joyner LG, Halenda PP (1951). The determination of pore volume and area distributions in porous substances.1. Computations from nitrogen isotherms. J. Am. Chem. Soc., 73: 373 - 380.

Beck JS, Vartuli JC, Roth WJ, Leonowicz ME, Kresge CT, Schmitt KD, Chu CT-W, Olson DH, Sheppard EW, McCullen SB, Higgins JB, Schlenker JL (1992). A new family of mesoporous molecular sieves prepared with liquid crystal templates. J. Am. Chem. Soc., 114: $10834-10843$.

Borisova D, Mohwald H, Shchukin DG (2011). Mesoporous silica nanoparticles for active corrosion protection. ACS NANO, 5(3): 1939 $-1946$.

Bottini M, Annibale FD, Magrini A, Cerignoli F, Arimura Y, Dawson MI, Bergamaschi E, Rosato N, Bergamaschi A, Mustelin T (2007). Quantum dot-doped silica nanoparticles as probes for targeting of Tlymphocytes. Int. J. Nanomed., 2: 227 - 233.

Brunauer S, Emmett PH, Teller E (1938). Adsorption of gases in multimolecular layers. J. Am. Chem. Soc., 60: 309 - 319.

Cauda V, Argyo C, Piercey DG, Bein T (2011). "Liquid-Phase Calcination" of Colloidal Mesoporous Silica Nanoparticles in HighBoiling Solvents. J. Am. Chem. Soc., 133: 6484 - 6486.

Cauda V, Muhlstein L, Onida B, Bein T (2009). Tuning drug uptake and release rates through different morphologies and pore diameters of confined mesoporous silica. Micropor. Mesopor. Mater., 118: 435 442.

Chen J, Wang W, Xu Y, Zhang X (2011). Slow-Release Formulation of a New Biological Pesticide, Pyoluteorin, with Mesoporous Silica. J. Agric. Food Chem., 59: 307 - 311.

Coleman NRB, Attard GS (2001). Ordered mesoporous silicas prepared from both micellar solutions and liquid crystal phases. Micropor. Mesopor. Mater., 44 - 45: 73 - 80.
Courtney RT, Daniel PF, Jae-Hyun L, Eunjoo C, MiHyeon C, Eun SK, Stoddart JF, Jeon-Soo S, JinWoo C, Jeffrey IZ (2010). Noninvasive Remote - Controlled Release of Drug Molecules in vitro using Magnetic Actuation of Mechanized Nanoparticles. J. Am. Chem. Soc., 132: 10623 -10625.

Cui D, Xu Q, Gu S, Shi J, Che X (2009). PAMAM-drug complex for delivering anticancer drug across blood-brain barrier in-vitro and invivo. Afr. J. Pharm. Pharmacol., 3(5): 227 - 233.

Feldmann C, Goesmann H (2010). Nanoparticulate Functional Materials. Angew. Chem. Int. Ed., 49: 1362 -1395.

Gao C, Qiu H, Zeng W, Sakamoto Y, Terasaki O, Sakamoto K, Chen Q, Che S (2006). Formation mechanism of anionic surfactant-templated mesoporous silica. Chem. Mater., 18: 3904 - 3914.

Gerion D, Herberg J, Bok R, Gjersing E, Ramon E, Maxwell R, Kurhanewicz J, Budinger TF, Gray JW, Shuman MA, Chen FF (2007). Paramagnetic silica-coated nano-crystals as an advanced MRI contrast agent. J. Phys. Chem., C 111: 12542 - 12551.

Graf C, Dembski S, Hofmann A, Ruehl E (2006). A general method for the controlled embedding of nanoparticles in silica colloids. Langmuir. 22: 5604 - 5610.

Gu J, Fan W, Shimojima A, Okubo T (2007). Organic-inorganic mesoporous nanocarriers integrated with biogenic ligands. Small, 3 : $1740-1744$

Hoffman F, Cornelius M, Morell J, Froba M (2006). Silica-Based Mesoporous Organic-Inorganic Hybrid Materials. Angew. Chem. Int. Ed., 45: 3216 - 3251.

Igor IS, Juan LV-E, Chia-Wen W, Victor S-YL (2008). Mesoporous silica nanoparticles as controlled release drug delivery and gene transfection carriers. Adv. Drug Deliv. Rev. 60: 1278 -1288.

Kobler J, Bein T (2008). Porous Thin Films of Functionalized Mesoporous Silica Nanoparticles. ACS NANO, 2(11): 2324 - 2330.

Kobler J, Moller K, Bein T (2008). Colloidal Suspensions of Functionalized Mesoporous Silica Nanoparticles. ACS NANO, 2(4): 791 - 799.

Leirose GDS, Cardoso MB (2011). Silica-Maltose Composites: Obtaining Drug Carrier Systems through Tailored Ultrastructural Nanoparticles. J. Pharm. Sci., 100(7): 2826 -2834.

Limnel T, Santos HA, Makila E, Heikkila T, Salonen J, Murzin DY, Kumar N, Laaksonen T, Peltonen L, Hirvonen J (2011). Drug Delivery Formulations of ordered Mesoporous Silica: Comparison of Three Drug loading methods. J. Pharm. Sci. Published online, D01.10.1002/jps.22577.

Liu R, Liao P, Liu J, Feng P (2011). Responsive polymer-coated mesoporous silica as $\mathrm{PH}$ - sensitive nanocarrier for controlled release. Langmuir, 27: 3095 - 3099.

Mbaraka IK, McGuire KJ, Shanks BH (2006). Acidic mesoporous silica for the catalytic conversion of fatty acids in beef tallow. Ind. Eng. Chem. Res. 45: 3022 - 3028.

Naik SP, Elangovan SP, Okubo T, Sokolov I (2007). Morphology control of mesoporous silica particles. J. Phys. Chem., C 111: 11168 11173.

Niu D, Ma Z, Li Y, Shi J (2010). Synthesis of core-shell structured dualmesoporous silica spheres with tunable pore size and controllable shell thickness. J. Am. Chem. Soc., 132: 15144 - 15147.

Nooney RI, Thirunavukkarasu D, Chen Y, Joseph R, Ostafin AE (2002). Synthesis of Nanoscale Mesoporous Silica Spheres with controlled particle size. Chemistry Mater., 14: 4721 - 4728.

Palmqvist AEC (2003). Synthesis of ordered mesoporous materials using surfactant liquid crystals or micellar solutions. Curr. Opin. Colloid. Interface Sci., 8: 145 -155.

Popat A, Hartono SB, Stahr F, Liu J, Qiao SZ, Lu GQ (2011). Mesoporous silica nanoparticles for bioadsorption, enzyme immobilization, and delivery carriers. Nanoscale. DOI: 10.1039/c1nr10224a.

Popovici RF, Seftel EM, Mihai GD, Popovici E, Voicu VA (2010). Controlled Drug Delivery System Based on Ordered Mesoporous Silica Matrices of Captopril as Angiotensin-Converting Enzyme Inhibitor Drug. J. Pharm. Sci., 100(2): 704 - 714.

Radin S, El-Bassyouni G, Vresilovic EJ, Schepers E, Ducheyne P (2004). In vivo tissue response to resorbable silica xerogels as controlled-release materials. Biomaterials, 26: 1043 - 1052.

Salonen J, Lehto VP, Laine E (1997). The room temperature oxidation 
of porous silicon. Appl. Surf. Sci., 120: 191 - 198.

Shen S-C, Ng WK, Chia L, Hu J, Tan RBH (2011). Physical state and dissolution of ibuprofen formulated by co-spray drying with mesoporous silica: Effect of pore and particle size. Int. J. Pharm., 410: $188-195$.

Shokri N, Javar HA, Fouladdel S, Khalaj A, Dinarvand R, Azizi E (2011). Preparation and characterization of crosslinked and non-crosslinked polycaprolactone fumarate (PCLF) NPs as carriers for doxorubicin HCl. Afr. J. Pharm. Pharmacol., 5(7): 797 - 805.

Sing KSW, Everett DH, Haul RAW, Moscou L, Pierotti RA, Rouquerol J, Siemieniewska T (1985). Reporting physisorption data for gas/solid systems with special reference to the determination of surface area and porosity. Pure Appl. Chem., 57: 603 - 619.

Slowing II, Trewyn BG, Lin VSY (2007). Mesoporous silica nanoparticles for intracellular delivery of membrane-impermeable proteins. J. Am. Chem. Soc., 129: 8845 - 8849.

Speybroeck MV, Mellaerts R, Mols R, Thi TD, Martens JA, Humbeeck JV, Annaert P, Mooter GV, Augustijns P (2010). Enhanced absorption of the poorly soluble drug fenofibrate by tuning its release rate from ordered mesoporous silica. Eur. J. Pharm. Sci., 41: 623 630.

Stober W, Fink A, Bohn E (1968). Controlled Growth of Monodisperse Silica Spheres in the Micron size range. J. Colloid Interface Sci., 26: 62-69.

Suteewong T, Sai H, Cohen R, Wang S, Bradbury M, Baird B, Gruner SM, Wiesner U (2011). Highly animated mesoporous silica nanoparticles with cubic pore structure. J. Am. Chem. Soc., 133: 172 -175 .

Thommes M, Kohn R, Froba M (2000). Sorption and Pore Condensation Behavior of Nitrogen, Argon, and Krypton in Mesoporous MCM-48 Silica Materials. J. Phys. Chem., B104: 7932794.

Vallet-Regı M (2009). Nanostructured mesoporous silica matrices in Nanomedicine. J. Int. Med., 267: 22 - 43.
Vivero-Escoto JL, Slowing II, Trewyn BG, Lin VS-Y (2010). Mesoporous Silica Nanoparticles for Intracellular Controlled Drug Delivery. Small. 6(18): 1952 - 1967.

Wen L-X, Li Z-Z, Zou H-K, Liu A-Q, Chen J-F (2005). Controlled release of avermectin from porous hollow silica nanoparticles. Pest Manag. Sci., 61: 583 - 590.

Yokoi T, Sakamoto Y, Terasaki O, Kubota Y, Okubo T, Tatsumi T (2006). Periodic Arrangement of Silica Nanospheres Assisted by Amino Acids. J. Am. Chem. Soc., 128: 13664 - 13665.

Yoo WC, Stein A (2011). Solvent effects on morphologies of mesoporous silica spheres prepared by pseudomorphic transformations. Chem. Mater., 23: 1761-1767.

Zhao XS, Lu GQ, Whittaker AK, Millar GJ, Zhu HY (1997). Comprehensive Study of Surface Chemistry of MCM-41 Using 29S CP/MAS NMR, FTIR, Pyridine-TPD, and TGA. J. Phys. Chem., B101: 6525-6531.

Zhu C-L, Lu C-H, Song X-Y, Yang H-H, Wang X-R (2011). Bioresponsive Controlled Release Using Mesoporous Silica Nanoparticles Capped with Aptamer-Based Molecular Gate. J. Am. Chem. Soc., 133: 1278 -1281.

Zhu MQ, Han JJ, Li Alexander DQ (2007). CdSe/CdS/SiO core/shell/shell nanoparticles. J. Nanosci. Nanotechnol., 7: 2343 2348. 